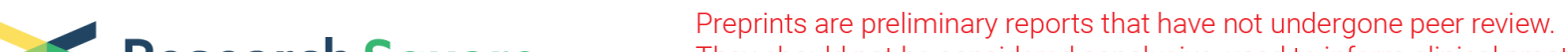 $\begin{array}{ll}\text { Research Square } & \text { They should not be considered conclusive, used to inform clinical practice, } \\ \text { or referenced by the media as validated information. }\end{array}$
}

\section{Genetic Analysis of Human Parainfluenza Virus Type 4-Associated with Severe Acute Respiratory Infection Among Children in Luohe City, Henan Province, China, During 2017-2018}

\section{Shanshan Zhou}

Inner Mongolia Medical University

\section{Naiying Mao}

Chinese Center for Disease Control and Prevention

\section{Yan Zhang}

Chinese Center for Disease Control and Prevention

Aili Cui

Chinese Center for Disease Control and Prevention

\section{Zhen Zhu}

Chinese Center for Disease Control and Prevention

\section{Ruiping Hu}

Inner Mongolia Medical University

Jin Xu

Henan Province CDC: Henan Province Center for Disease Control and Prevention

Wenbo Xu ( $\square$ wenbo_xu63@126.com)

Chinese Center for Disease Control and Prevention https://orcid.org/0000-0001-5911-8837

\section{Research Article}

Keywords: Nasopharyngeal Aspirates, Genetic Analysis, Epidemiological and Clinical Characteristics

Posted Date: April 5th, 2021

DOI: https://doi.org/10.21203/rs.3.rs-363959/v1

License: (c) (i) This work is licensed under a Creative Commons Attribution 4.0 International License. Read Full License

Version of Record: A version of this preprint was published at Archives of Virology on July 6th, 2021. See the published version at https://doi.org/10.1007/s00705-021-05154-3. 


\section{Abstract}

During 2017-2018, Nasopharyngeal aspirates (NPAs) from 627 hospitalized patients with SARI at Luohe Center Hospital were tested by RT-PCR for human parainfluenza virus 4(HPIV-4). 14 (2.2\%) of 627 samples were positive for HPIV-4. The complete nucleotide sequence of the HN gene from 9 positive samples was amplified and sequenced successfully. Genetic analysis showed that the HPIV-4 strains circulating in Luohe city are more closely related to HPIV-4A. Our study indicated that there were multiple lineages of HPIV-4 circulating in Henan Province in China during the study period, which will improve our understanding of the epidemiological and clinical characteristics of HPIV-4.

\section{Main Text}

Human parainfluenza viruses (HPIVs), belonging to the family Paramyxoviridae, are enveloped, negative, single-stranded RNA viruses [1, 2]. Based on genetic and antigenic variation, HPIVs have been divided into four serotypes into 2 different genera: Respirovirus (HPIV-1 and HPIV-3) and Rubulavirus (HPIV-2 and HPIV-4) [3, 4]. Globally, HPIVs account for a significant proportion of acute respiratory infections (ARIs) among children under the age of 5 years [5, 6]. HPIV-4 was first identified in 1959 by Johnson et al. [7] and was formerly associated with mild respiratory illness in young people. However, recent studies indicate that it can cause more severe infections, such as pneumonia and bronchiolitis, in children and elderly individuals [8-12] and even in immunocompetent individuals and critically ill patients [13-15].

HPIV-4 is subdivided into two subtypes, HPIV-4A and HPIV-4B, based on hemagglutination inhibition and neutralization tests [16]. However, although studies associated with HPIV-4 infection have increased globally, possibly due to improved and increased diagnostic testing, the molecular characteristics of regional and global circulating HPIV-4 strains have not been fully elucidated.

In this study, 627 nasopharyngeal aspirates (NPAs) were collected and screened for HPIV-4 infection from hospitalized patients with severe acute respiratory infection (SARI) in Luohe city, Henan Province, China, during 2017-2018. Informed consent was obtained from patients or their guardians for the donation of their samples involved in this study. This study was approved by the second session of the Ethics Review Committee of the National Institute for Viral Disease Control and Prevention (IVDC) of the Center for Disease Control and Prevention (CDC) in China, and the methods were conducted according to the guidelines. SARI cases were identified according to the sentinel surveillance program for hospitalized SARI cases in China (http://www.gov.cn/zwgk/2011-02/11/content_1801649.htm).

The samples were transported to the IVDC of the China CDC under cold chain for further identification. Specimens were stored in sterile minimal medium at -20 or $-80^{\circ} \mathrm{C}$ pending molecular analysis. All methods were performed in accordance with the relevant guidelines and regulations.

Viral RNA was extracted from NPAs samples using a QIAgen viral RNA mini kit(QIAgen, Valencia, CA, USA), and HPIV-4 positive samples were screened by one-step real-time RT-PCR using a PrimeScript ${ }^{\mathrm{TM}}$ RT- $^{-}$ PCR Kit (Takara Biotechnology Dalian, China, cat: DRR064A) as described previously [17]. The complete 
hemagglutinin-neuraminidase (HN) gene (1740 nt) of HPIV-4 was amplified by nested RT-PCR for genotyping HPIV-4. The first round of RT-PCR was performed using the following in-house designed primer pair: forward primer, 5'- ATAGGGGGGAACRCACTTCTCAGC-3'; reverse primer, 5'-

GGCRGRTTGTTTRTYGAGGACC-3'. The nested PCR was carried out with the inner primers forward primer, 5'-AACAATCCAGARRGACRTCACATCAA-3' and reverse primer, 5'- TCTTTCAGTGGATGGTTGAGGA-3'. The RT-PCR products were purified for sequencing using a QIAgen quick gel extraction kit (QIAgen, Hilden, Germany), and the amplicons were sequenced on an ABI PRISM 3100 DNA Sequencer (PerkinElmer, Beijing, China). Nucleotide sequences were assembled and edited using Sequencher (Gene Codes Corporation, Ann Arbor, MI, USA). The sequences of HPIV-4 in our study have been submitted to GenBank (the accession numbers are MT681670-MT681678).

Pairwise distances of nucleotide and deduced amino acid sequences were aligned with 43 HPIV-4 strains from GenBank using the ClustalW algorithm implemented in MEGA software version 7.0. Phylogenetic analyses based on complete HN gene nucleotide sequences were performed (MEGA version 7.0) by the maximum likelihood method using Kimura 2-parameters as the substitution model, with statistical significance of phylogenies estimated by bootstrap analysis with 1,000 replicates.

From October 2017 to December 2018, a total of 627 NPAs from inpatients (391 males and 236 females) who met the SARI case definition were collected from Luohe Central Hospital in Henan Province. The hospitalized patients with SARI were aged from 0 to 91 years, among which children under 6 years old accounted for $78.0 \%$ (489/627). The median age was 7.9 years.

Of the 627 patients, $14(2.2 \%, 95 \% \mathrm{Cl} 1.1-3.4)$ patients were positive for HPIV-4 by real-time RT-PCR. The cycle threshold (CT) values for the HPIV-4-positive samples ranged from 25 to 35 . Most of the CT values of the positive samples ranged from 29 to 33 . The ratio of males to females was 1.7:1. Among the 14 patients (median 2.5 years) with determined HPIV-4, all of them were younger than 7 years old, and $50 \%$ ranged from 0 to 3 years of age (Table 1). Among the pediatric patients, 10 (71.4\%) were diagnosed with bronchopneumonia, 3 (21.4\%) with bronchiolitis, and 1 (7.1\%) with mucocutaneous lymph node syndrome (MCLS).

The seasonality of HPIV-4 was observed throughout the study period. The positive rates of HPIV-4 in spring, summer, autumn, and winter were $1.2 \%$ (1/85), $17.5 \%$ (10/57), 0.5\% (1/199), and 0.7\% (2/286), respectively (Figure 1). The highest detection rates of HPIV-4 were found in summer, and the detection rate of HPIV-4 varied significantly between seasons $\left(X^{2}=67.456, P=0.000\right)$.

The complete HN gene sequences of 9 HPIV-4 (CT values between 25 and 33) isolates amplified from 14 HPIV-4-positive specimens were analyzed. The HN gene failed to amplify from the remaining 5 HPIV-4positive specimens, which had CT values ranging from 27 to 35 in the real-time RT-PCR. This could be due to mismatches in the primer-binding sites or low viral load.

All HPIV-4 HN gene sequences available in GenBank (43 sequences) were downloaded and aligned with the 9 sequences obtained in this study. Among the 9 HPIV-4 HN gene sequences, 7 sequences clustered 
with the HPIV-4A reference strains, and the remaining 2 clustered with the HPIV-4B reference strains (Figure 2). By convention, the prototype of HPIV-4, which was isolated in Japan in 1959, formed a single

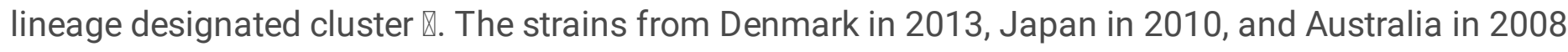

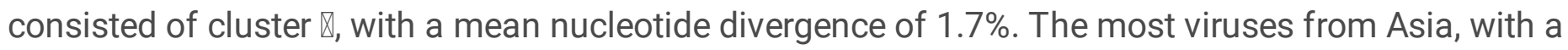

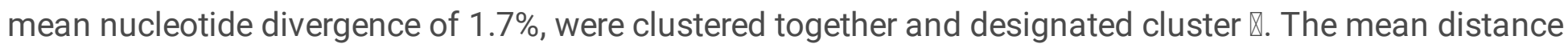
between the three clusters is $5.4 \%$ and is larger than the mean distance within the three clusters $(1.7 \%)$, in reference to a report that described phylogenetic analysis of HPIV-3 [18]. Cluster III strains were further grouped into four lineages (lineages 1, 2, 3 and 4) with 1.8-2.7\% nucleotide divergence. In our study, 6 HPIV-4A strains belonged to the lineage 4 group together with the previously reported 4 strains from Japan. The remaining HPIV-4A strain was placed into lineage 3 with 7 strains from Japan. Additionally, 2 strains found in this study were identified as HPIV-4B, which has been rarely reported in recent decades.

The divergence between the 7 HPIV-4A strains in our study and the prototype strain M-25 was approximately $6.6 \%-6.9 \%$ and $10.1 \%-11.0 \%$ in the nucleotide and amino acid sequences, respectively. The two HPIV-4B isolates revealed $4.9 \%-5.0 \%$ nucleotide divergence and $8.0 \%-8.2 \%$ amino acid divergence compared with the prototype strain. Notably, the length of the HN gene sequence (1740 nt) in our 9 strains is longer than that of the prototype strain ( $1725 \mathrm{nt}$ ) by 15 nucleotides ( 5 amino acids) at the carboxy-terminus. Compared with other strains, the divergence of nucleotides and amino acids among HPIV-4A was 1.0\%-3.7\% and 0.3-3.4\%, respectively. Among HPIV-4B, it was 0.5-4.9\% and 0.5-8.4\%, respectively. Briefly, there are two common subtypes of HPIV-4 in China, HPIV-4A and HPIV-4B, of which

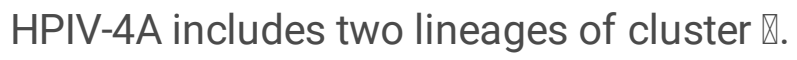

In the present study, 14 samples positive for HPIV-4 from 627 (2.2\%) SARI patients collected between October 2017 and December 2018 were analyzed to determine the epidemiology of HPIV-4 in Luohe city, Henan Province, China, by using q-RT-PCR. All patients with HPIV-4 infection were less than 7 years old, $57 \%$ of who were younger than 3 years, indicating that children of this age group are the main HPIV-4susceptible population. This result was similar to the results of previous reports. The positive rate of HPIV-4 was $1.2 \%$ among 0.5 -month-old to 16 -year-old patients, especially 3 -5-year-old children in Beijing, China, and the median patient age was 4.1 years old in Colorado between 2009 and 2012 [19, 20]. Therefore, HPIV-4 may be gradually becoming an important pathogen that causes SARI in children, which is more severe than previously thought.

In our study, HPIV-4 infections occurred during summer and autumn, especially in summer, which is inconsistent with published studies from other countries. In Japan, Abiko et al. [8] described an outbreak of HPIV-4 infections during the 2011-2012 winter season. The same result was reported in Canada during 2004-2005 [21]. This finding may be attributed to different geographical regions and research times. However, as the number of positive samples is limited in this study, a large-scale investigation of the HPIV-4-positive rate is needed to understand the seasonal patterns of HPIV-4. Additionally, considering that only strains from hospitalized patients were analyzed in this study, the possibility that other underestimated strains could be circulating in the general population cannot be ruled out. 
Previous studies reported that HPIV-4 strains were detected at different times in different regions [21-23]. In our study, we characterized the HN gene from HPIV-4-positive samples from patients with SARI and further divided all of the HPIV-4A strains into three clusters. This study is the first report that has described the phylogeny of HPIV-4 based on the complete HN sequence. Compared with the HPIV-4 prototype strain M-25, 9 strains in our study, including other strains from GenBank, demonstrated significantly different characteristics. A five amino acid insert in the HN protein of Luohe strains may result in changes in the antigen-binding site and have a subsequently severe influence on viral replication and transmission. This finding also indicates that the viruses are probably evolving.

Phylogenetic analysis revealed 5 (Henan-SA20180251/247/303/268/292) of 7 HPIV-4A strains that might share a chain of transmission. The remaining two HPIV-4A strains were similar to the Japanese strains between 2010 and 2011. As the phylogenetic tree shows, it is suggested that the domestic HPIV-

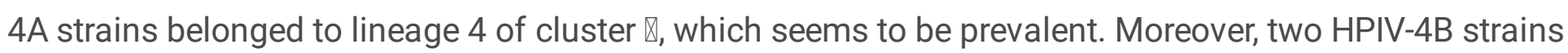
were associated with three USA HPIV-4B strains isolated during 2015-2016. This result reveals that the HPIV-4 strains were undergoing global circulation and more closely related to HPIV-4A than HPIV-4B, based on the HN gene sequences in our study. However, additional sequences are needed to expand the dataset, and additional studies are required to confirm the relative importance of HPIV-4A and HPIV-4B.

In conclusion, we reported the HN gene sequences of 9 HPIV-4 strains isolated from SARI patients. The divergence among HPIV-4 strains indicated that these viruses have circulated in the environment for many years and have undergone evolution. To better recognize its clinical importance and seasonal patterns, HPIV-4 should be included in the routine panels of respiratory virus detection, although most clinical laboratories do not screen for HPIV-4 [23, 24]. This report provides valuable information about HPIV-4 isolates to help prevent HPIV-related respiratory diseases.

\section{Declarations}

\section{Acknowledgments}

The authors are grateful to the clinicians of Luohe Central Hospital for assisting with sample collection.

\section{Authors' contributions}

SSZ, NYM and WBX prepared the manuscript. RPH, JX and WBX designed and coordinated the study. YZ, ALC and ZZ collected the specimens and performed the experiments. SSZ, NYM, YZ, ALC, ZZ and WBX performed the data analysis. All authors read and approved the final manuscript.

\section{Funding}

Funding was obtained from the Key Technologies R\&D Program of the National Ministry of Science (Grant No. 2018ZX10713002 and 2018ZX10713001-003).

\section{Data availability}


All data included in this study are available upon request from the corresponding author.

\section{Conflict of interest}

The authors declare that they have no competing interests.

\section{References}

1. Vainionpaa R, Hyypia T (1994) Biology of parainfluenza viruses. Clin Microbiol Rev 7:265-275

2. Branche AR, Falsey AR (2016) Parainfluenza virus infection. Semin Respir Crit Care Med 37:538-554

3. Henrickson KJ (2003) Parainfluenza viruses. Clin Microbiol Rev 16:242-264

4. Park KS, Yang MH, Lee CK, Song KJ (2014) Genetic analysis of human parainfluenza viruses circulating in Korea, 2006. J Med Virol 86:1041-1047

5. Pecchini R, Berezin EN, Souza MC, de Andrade Vaz-de-Lima L, Sato N, Salgado M et al. (2015) Parainfluenza virus as a cause of acute respiratory infection in hospitalized children. Braz $\mathrm{J}$ Infect Dis 19:358-362

6. Weinberg GA (2006) Parainfluenza viruses: an underappreciated cause of pediatric respiratory morbidity. Pediatr Infect Dis J 25:447-448

7. Johnson KM, Chanock RM, Cook MK, Huebner RJ (1960) Studies of a new human hemadsorption virus. I. Isolation, properties and characterization. Am J Hyg 71:81-92

8. Abiko C, Mizuta K, Aoki Y, Ikeda T, Itagaki T, Noda M et al. (2013) An outbreak of parainfluenza virus type 4 infections among children with acute respiratory infections during the 2011-2012 winter season in Yamagata, Japan. Jpn J Infect Dis 66:76-78

9. Fairchok MP, Martin ET, Kuypers J, Englund JA (2011) A prospective study of parainfluenza virus type 4 infections in children attending daycare. Pediatr Infect Dis J 30:714-716

10. Russell E, Yang A, Tardrew S, Ison MG (2019) Parainfluenza virus in hospitalized adults: a 7-year retrospective study. Clin Infect Dis 68:298-305

11. Steffens A, Finelli L, Whitaker B, Fowlkes A (2016) Population-based surveillance for medically attended human parainfluenza viruses from the influenza incidence surveillance project, 2010-2014. Pediatr Infect Dis J 35:717-722

12. Pan $Y$, Zhang $Y$, Shi W, Peng $X$, Cui S, Zhang D et al. (2017) Human parainfluenza virus infection in severe acute respiratory infection cases in Beijing, 2014-2016: A molecular epidemiological study. Influenza Other Respir Viruses 11:564-568

13. Miall F, Rye A, Fraser M, Hunter A, Snowden JA (2002) Human parainfluenza type 4 infection: a case report highlighting pathogenicity and difficulties in rapid diagnosis in the post-transplant setting. Bone Marrow Transpl 29:541-542

14. Pawelczyk M, Kowalski ML (2017) The role of human parainfluenza virus infections in the immunopathology of the respiratory tract. Curr Allergy Asthma Rep 17:16 
15. Essa S, Al-Tawalah H, AlShamali S, Al-Nakib W (2017) The potential influence of human parainfluenza viruses detected during hospitalization among critically ill patients in Kuwait, 20132015. Virol J 14:19

16. Canchola J, Vargosko AJ, Kim HW, Parrott RH, Christmas E, Jeffries B et al. (1964) Antigenic variation among newly isolated strains of parainfluenza type 4 virus. Am J Hyg 79:357-364

17. Jansen RR, Schinkel J, Koekkoek S, Pajkrt D, Beld M, de Jong MD et al. (2011) Development and evaluation of a four-tube real time multiplex PCR assay covering fourteen respiratory viruses, and comparison to its corresponding single target counterparts. J Clin Virol 51:179-185

18. Mao N, Ji Y, Xie Z, Wang H, Wang H, An J et al. (2012) Human parainfluenza virus-associated respiratory tract infection among children and genetic analysis of HPIV-3 strains in Beijing, China. PLoS One 7:e43893

19. Frost HM, Robinson CC, Dominguez SR (2014) Epidemiology and clinical presentation of parainfluenza type 4 in children: a 3-year comparative study to parainfluenza types 1-3. J Infect Dis 209:695-702

20. Ren L, Gonzalez R, Xie Z, Xiong Z, Liu C, Xiang Z et al. (2011) Human parainfluenza virus type 4 infection in Chinese children with lower respiratory tract infections: a comparison study. J Clin Virol 51:209-212

21. Vachon ML, Dionne N, Leblanc E, Moisan D, Bergeron MG, Boivin G (2006) Human parainfluenza type 4 infections, Canada. Emerg Infect Dis 12:1755-1758

22. Billaud G, Morfin F, Vabret A, Boucher A, Gillet Y, Crassard N et al. (2005) Human parainfluenza virus type 4 infections: a report of 20 cases from 1998 to 2002. J Clin Virol 34:48-51

23. Lau SK, Li KS, Chau KY, So LY, Lee RA, Lau YL et al. (2009) Clinical and molecular epidemiology of human parainfluenza virus 4 infections in hong kong: subtype 4B as common as subtype 4A. J Clin Microbiol 47:1549-1552

24. Lau SK, To WK, Tse PW, Chan AK, Woo PC, Tsoi HW et al. (2005) Human parainfluenza virus 4 outbreak and the role of diagnostic tests. J Clin Microbiol 43:4515-4521

\section{Table}

Table 1. Sex and age distribution of HPIV-4-positive patients 


\begin{tabular}{|c|c|c|}
\hline & Number of total specimens & Number positive for HPIV-4 (\%) \\
\hline \multicolumn{3}{|l|}{ Sex } \\
\hline Males & 391 & $8(2.0 \%)$ \\
\hline Females & 236 & $6(2.5 \%)$ \\
\hline \multicolumn{3}{|c|}{ Age group (years) } \\
\hline$<1$ & 77 & $1(1.3 \%)$ \\
\hline$\geq 1$ to $<3$ & 238 & $7(2.9 \%)$ \\
\hline$\geq 3$ to $<6$ & 174 & $5(2.9 \%)$ \\
\hline$\geq 6$ to $<14$ & 67 & $1(1.5 \%)$ \\
\hline$\geq 14$ & 71 & 0 \\
\hline Total & 627 & $14(2.2 \%)$ \\
\hline
\end{tabular}

Figures

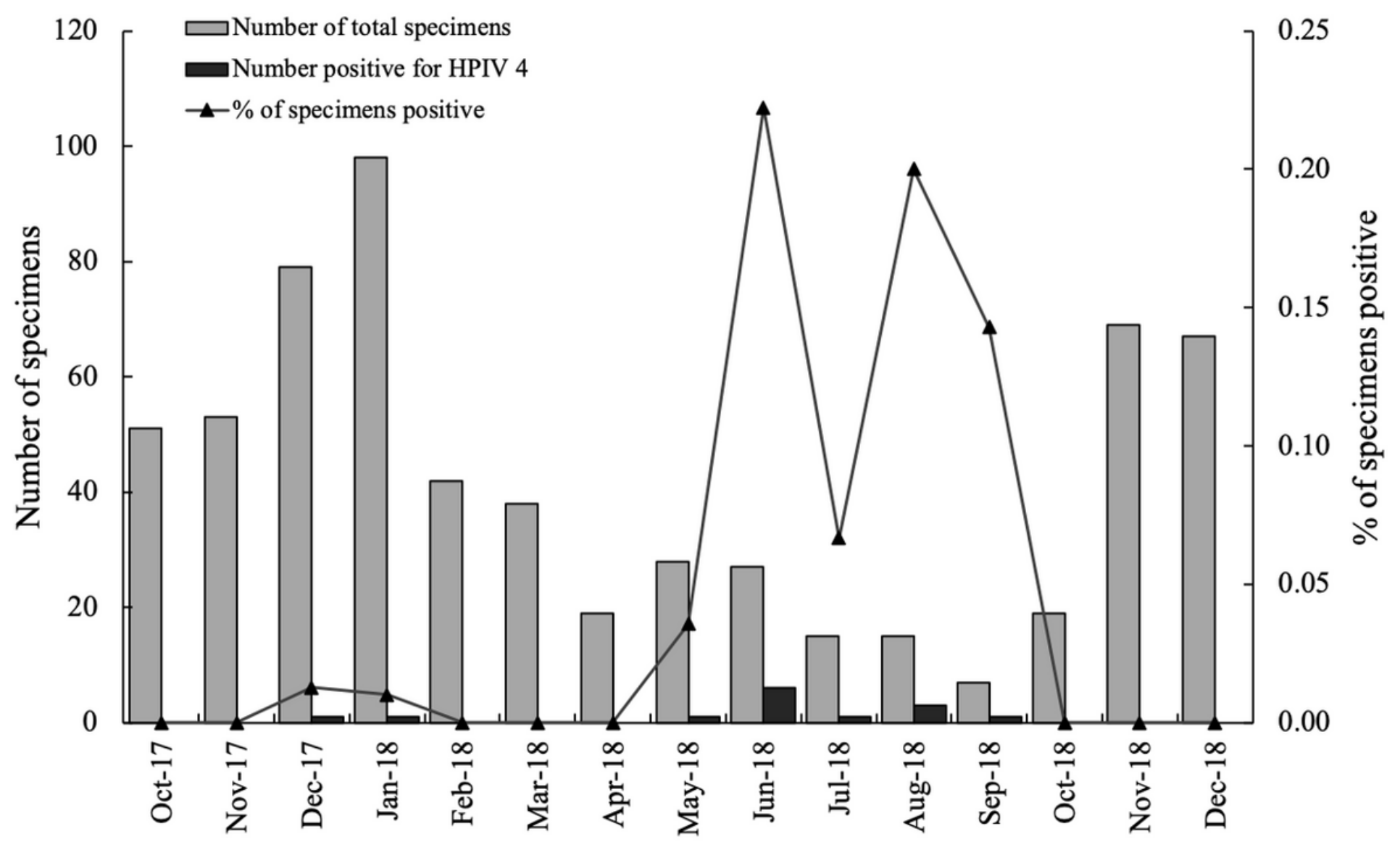

Figure 1 
Monthly distribution of human parainfluenza virus 4 (HPIV-4) infections

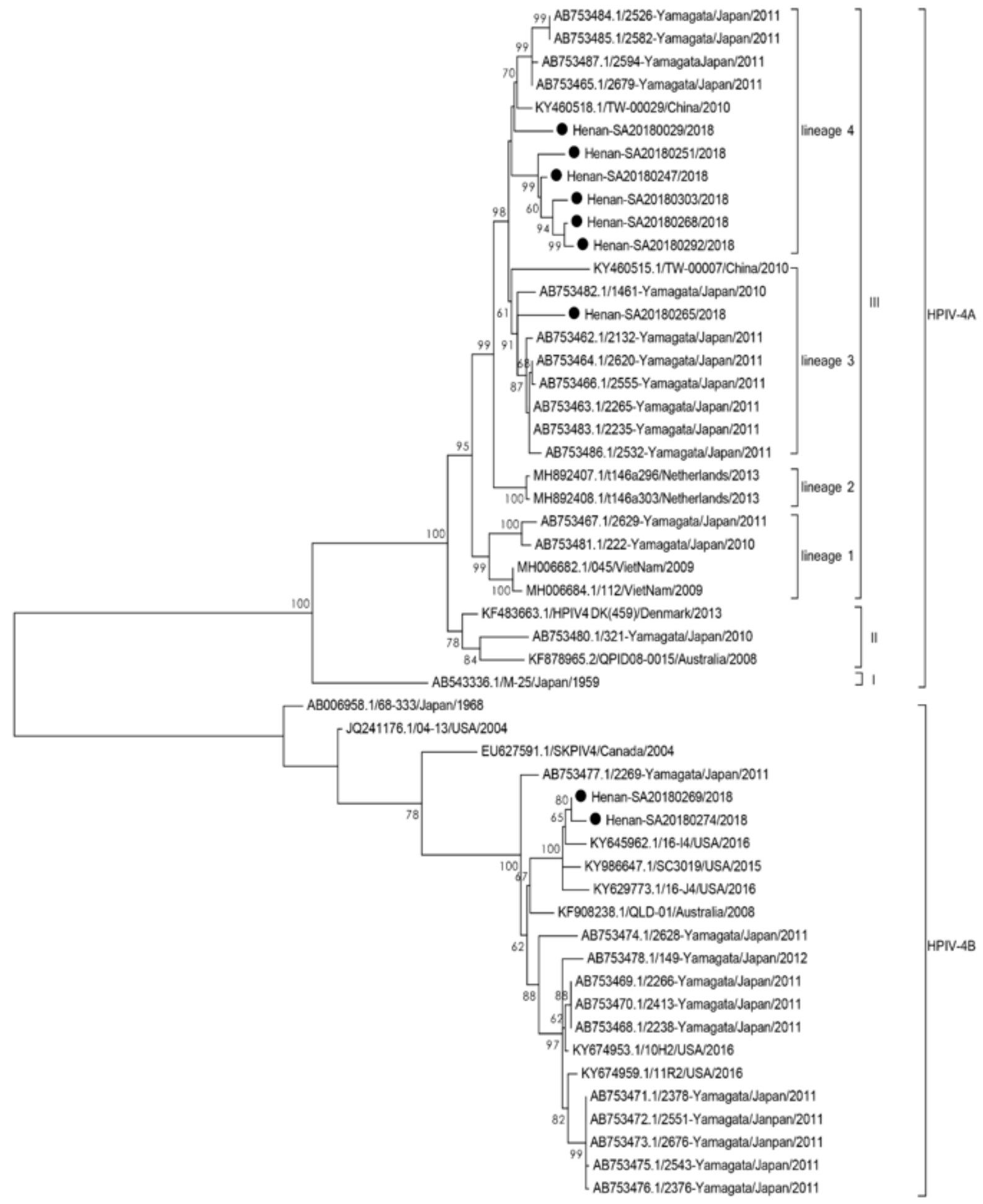

0.02

Figure 2

Phylogenetic tree based on complete HN gene nucleotide sequences of HPIV-4 strains

Supplementary Files 
This is a list of supplementary files associated with this preprint. Click to download.

- Submission.txt 